\title{
Le rubriche in volgare del codice 7-1-52 della biblioteca Colombina di Siviglia
}

\author{
Alina Zvonareva \\ Università degli Studi di Padova
}

\begin{abstract}
Sommario:
Il presente contributo propone uno studio storico-linguistico delle rubriche in volgare del ms. 7-1-52 della biblioteca Colombina di Siviglia. Si tratta di un codice francescano trecentesco trascritto in Italia settentrionale, contenente testi di carattere didattico-religioso e contraddistinto da uno spiccato ibridismo linguistico: nel corpus di riferimento si riscontrano tratti veronesi, veneziani o venezianeggianti, padovani ed emiliani. Il quadro dei fenomeni linguistici che emerge dalle rubriche viene confrontato nell'articolo con le peculiarità linguistiche che presenta il testo principale dei componimenti tràditi dal manoscritto; sulla base dello studio svolto si formulano delle ipotesi riguardo la datazione e la localizzazione delle rubriche. Inoltre, tale confronto permette di mettere il materiale linguistico in relazione con il problema della trasmissione e ricezione dei testi copiati. In particolare, viene postulato che i titoli del nostro codice rappresentino un volgarizzamento delle rubriche latine dell'archetipo perduto, e a tale proposito vengono esaminati anche gli altri testimoni manoscritti dei testi del corpus. Ci si interroga anche sul valore stilistico di tratti latineggianti, toscaneggianti e marcatamente locali, nonché su cosa significhi il fatto stesso di volgere le rubriche in un volgare settentrionale.
\end{abstract}

\section{Parole chiave:}

Tradizione manoscritta, rubriche, ibridismo linguistico, veneto antico, emiliano antico, letteratura religiosa, volgarizzamento.

\begin{abstract}
:
This article presents a historical-linguistic study on the vernacular rubrics of Seville Colombina manuscript 7-1-52. This is a 14th-century Franciscan codex copied in Northern Italy, containing didactic-religious texts and marked by linguistic hybridism: the reference corpus shows Veronese, Venetian or Venetian-like, Paduan and Emilian features. The article compares the linguistic phenomena present in the rubrics to the linguistic peculiarities of the main texts copied in the manuscript; on such basis, some hypotheses are formulated concerning the dating and the localization of the rubrics. Furthermore, such comparison allows to establish a connection between the linguistic material and the problem of transmission and reception of the copied texts. In particular, the chapter titles in our codex are postulated to be a volgarizzamento (vernacular translation) of the Latin rubrics in the lost archetype; with such purpose, the other manuscript witnesses of the texts in our corpus are examined. Other issues taken into consideration are the stylistic value of Latin-like, Tuscan-like and markedly local features, and the possible reasons for translating the rubrics into a Northern Italian vernacular language.
\end{abstract}


Key words:

Manuscript tradition, rubrics, linguistic hybridism, old Venetian, old Emilian, religious literature, volgarizzamento (vernacular translation).

\section{Premessa}

Il codice 7-1-52 della biblioteca Colombina di Siviglia, databile alla seconda metà del Trecento, contiene dieci componimenti di carattere didattico-religioso in antico veneto e toscano ${ }^{1}$. All'interno di questa raccolta di testi si riscontra un'altra raccolta più piccola, racchiusa nelle cc. 1r-41v e individuabile sulla base dei contenuti (i testi trascritti in questa sezione del codice sono tutti di matrice francescana, formano un'unità stilisticamente omogenea e presentano, a livello testuale, numerosi richiami interni), dei dati codicologici e paleografici (la mise en page, la fascicolazione e la posizione dei fogli rimasti bianchi permettono di suddividere il codice in più unità e di individuare una corrispondenza tra le unità codicologiche e di contenuto), della lingua (il quadro dei fenomeni linguistici riscontrabili alle cc. 1r-41v è diverso da quello che presentano gli altri testi tramandati dal manoscritto in questione). Inoltre, è opportuno mettere lo studio del codice in relazione con l'aspetto ecdotico, ovvero la tradizione manoscritta di singoli testi. Un dato importante è costituito dai rapporti del nostro codice con un altro testimone: i sette testi trascritti alle cc. 1r$41 \mathrm{v}$ del ms. colombino 7-1-52 sono tràditi anche dalle cc. 50r-111r del ms. 4744 (it. Zanetti XIII) della biblioteca Marciana di Venezia ${ }^{2}$ I componimenti si leggono nello stesso ordine in entrambi i codici, il che dimostra che la raccolta doveva esistere già nell'archetipo a cui risalgono i manoscritti di Siviglia (d'ora in avanti S) e di Venezia (d'ora in avanti V); una serie di errori congiuntivi confermano l'esistenza di un archetipo comune dei due testimoni. Il confronto con la redazione di V risulta utile per lo studio del codice S sotto vari aspetti, soprattutto quello storico-linguistico (il materiale fornito da $\mathrm{V}$ aiuta a interpretare una serie di fenomeni fono-morfologici

1 Il codice tramanda i seguenti testi: De Jerusalem celesti, De Babilonia infernali, Dell'amore di Gesù, Del Giudizio Universale, Della caducità della vita umana (sermoni in versi), Lodi della Vergine, Preghiera alla Vergine e alla Santissima Trinità (preghiere di carattere dossologico), la Leggenda di santa Margherita (un testo agiografico), una 'passione' di Cristo, la Lamentatio beate Virginis di Enselmino da Montebelluna (un 'pianto della Vergine'). I titoli delle prime sette opere menzionate sono convenzionali e risalgono ad Adolfo Mussafia, il primo editore dei componimenti (Mussafia 1864).

2 Mussafia (1864) editò tutto il corpus sulla base del solo codice V. I componimenti De Jerusalem celesti e De Babilonia infernali erano stati pubblicati, in un'edizione poco affidabile, da Ozanam (1859); dopo l'edizione Mussafia i due testi sono stati riediti da Emilio Barana (1921), Esther May (1930) e Gianfranco Contini (1960: 625-650) sulla base di tutta la tradizione manoscritta pervenuta (compresi i codici di Udine e di Oxford, chiamati in causa anche nel presente contributo); il componimento Della caducità della vita umana è stato pubblicato da Contini e Romano Broggini (Contini 1960: 653-666) sulla base dei codici V e S. 
registrati in S) e quello critico-testuale (per l'allestimento dell'edizione interpretativa della redazione di $\mathrm{S}^{3}$ si è tenuto conto anche delle lezioni del codice $\mathrm{V}$ ).

All'interno della raccolta di sette componimenti si distinguono i primi due testi, gli unici di cui si conosce il nome dell'autore, Giacomino da Verona (mentre i restanti componimenti del corpus sono anonimi), e di cui ci sono pervenuti, oltre a V e S, altri due testimoni: il codice Qt. XIII. I. 26 della biblioteca Arcivescovile di Udine (cc. 40r-50v) e il codice Canoniciano Italiano 48 della biblioteca Bodleiana di Oxford (cc. 1r-5v, dove è trascritto solo il primo dei due componimenti, con lacune). Per la parte contenente i poemetti di Giacomino da Verona è opportuno, dunque, confrontare $\mathrm{S}$ anche con i codici di Udine (d'ora in avanti U) e di Oxford (d'ora in avanti $\mathrm{O}$ ).

Il manoscritto $\mathrm{S}$ finora non è mai stato sistematicamente esaminato dal punto di vista storico-linguistico ${ }^{4}$; eppure la sua veste linguistica merita di essere studiata in quanto presenta una serie di fenomeni piuttosto singolari e fa trasparire alcuni aspetti della diffusione della raccolta: essendo un testimone piuttosto tardo, distante almeno qualche decennio dagli originali dei testi che tramanda e trascritto da amanuensi che avevano poca dimestichezza con la lingua dell'originale (il veronese di fine Duecento - inizio Trecento) $)^{5}$, S permette di ricostruire dei passaggi del testo - legati a delle copie intermedie eseguite in città diverse - attraverso più aree municipali dell'Italia settentrionale. Nell'insieme dei tratti linguistici registrati in S si individuano uno strato veronese (è sicuramente lo strato più antico, riconducibile all'ascendente da cui deriva tutta la trafila di copie), uno strato veneto posteriore all'archetipo, poco

3 Fornisco un'edizione interpretativa delle carte 1r-41v di S nella mia tesi di dottorato.

4 Un tentativo, sulla base delle carte contenenti i poemetti di Giacomino da Verona, era stato intrapreso in May 1930: 58-61; tuttavia, pur senza negare i meriti di questo lavoro, bisogna ammettere che lo studio linguistico proposto non può essere definito esaustivo, e parecchie conclusioni a cui giunge sono piuttosto discutibili.

5 Il valore di V consiste invece nell'essere il manoscritto più vicino all'archetipo e all'originale sia dal punto di vista cronologico sia da quello linguistico: è databile agli inizi del Trecento (Mussafia 1864: 113; Frati-Segarizzi 1909: 12; Barana 1921: 32), e localizzabile a Verona; il codice tramanda, oltre alla nostra raccolta di componimenti didattico-religiosi in versi, altri testi importanti per lo studio del veronese antico, come la redazione veronese della Leggenda di Santa Margherita, un Contrasto tra Cristo e il diavolo (Verlato 2002), un volgarizzamento del Transitus Pseudo-Josephi de Arimathea (Cornagliotti 1979), ecc. I codici U e O dal punto di vista dell'aspetto formale si allontanano dall'archetipo veronese, ma in misura minore rispetto a S. Su alcune caratteristiche linguistiche di U e O si soffermano Barana (1921: 23-29) e May (1930: 49-58); May localizza O in Emilia e U a Grado, Barana invece assegna $U$ a Treviso e definisce $\mathrm{O}$ scorretto e privo di marcati tratti dialettali e quindi rinuncia a stabilire la sua provenienza; per quel che mi riguarda, trovo convincenti la maggior parte delle argomentazioni con le quali May riconduce $\mathrm{O}$ alla zona emiliana; quanto a $\mathrm{U}$, la sua provenienza veneto-orientale (area trevigiana) mi sembra plausibile (ma probabilmente non è il caso di insistere su Grado). 
omogeneo ma riconducibile al veneziano (o piuttosto alla koiné venezianeggiante) e al veneto centrale (padovano), uno strato emiliano (probabilmente bolognese) e la patina toscana, verosimilmente veicolata da copie di aree veneta ed emiliana ${ }^{6}$.

Nel presente contributo si esaminano le rubriche in volgare che in S precedono ciascuno dei componimenti del corpus definito sopra. Tali rubriche sono interessanti dal punto di vista linguistico, se messe a confronto con il testo principale dei componimenti trascritti in $\mathrm{S}$.

Di tutti e quattro i codici che trasmettono i testi che qui ci interessano solo in $\mathrm{S}$ tutti i componimenti sono corredati da titoli. In $\mathrm{V}$ le rubriche accompagnano solo i due poemetti di Giacomino da Verona, mentre i manoscritti $\mathrm{U}$ e $\mathrm{O}$ trascrivono questi testi senza titolo. Le due rubriche di V sono in latino: De Jerusalem celesti et de pulcritudine eius et beatitudine et gaudia sanctorum (50r) e De Babilonia civitate infernali et eius turpitudine et quantis penis peccatores puniantur incessanter (57r).

Riportiamo tutte le rubriche di S in trascrizione interpretativa (le abbreviazioni sono sciolte tra parentesi tonde; le note tironiane simili a 7 sono trascritte come et; le integrazioni per congettura sono poste tra parentesi quadre; le cruces nella rubrica $\mathrm{D}$ segnalano un errore la cui correzione è riportata subito sotto; abbiamo introdotto le maiuscole e i segni diacritici e abbiamo regolarizzato la separazione delle parole secondo l'uso moderno; le particolarità grafiche del manoscritto sono conservate):

$\mathrm{A}^{7}$ (1r): Di Jerusalen celeste e de lla belleçça di quella e d(e) lla beatitudine e allegreçe de' santi.

B (5v): Di Babillonia cità i(n)fernala e della bructeça di quella e di quanti pecadi sono se(n)pre ponidi li pecatori.

C (11r): De ll'amore de Cristo quanto sia suave e dolçe e della operacion che fa i(n) del core di colui lo qual l'ama co(n) la ferma mente.

D (17r): Del conforto che fa l'anima al corpo e de l'ultimo giudicio di Dio in del qual ricieverà ciascu(n) secondo l'opra ch'avrà †fatè $\dagger$.

fate] fata

6 Dell'ibridismo linguistico di S mi sono occupata in Zvonareva 2012.

7 Le sigle $(\mathrm{A}=$ De Jerusalem celesti, $\mathrm{B}=$ De Babilonia infernali, $\mathrm{C}=$ Dell'amore di Gesù, $\mathrm{D}=$ Del Giudizio Universale, $\mathrm{E}=$ Della caducità della vita umana, $\mathrm{F}=$ Lodi della Vergine, $\mathrm{G}=$ Preghiera alla Vergine e alla Santissima Trinità) sono usate anche laddove si indicano le fonti degli esempi tratti dal testo dei componimenti secondo l'edizione interpretativa del codice $\mathrm{S}$ che fornisco nella mia tesi di dottorato; ogni riferimento è composto dalla sigla del componimento e dal numero del verso. 
E (23v): Del pia(n)golente nasimento de l'omo et de lla sua misera vita i(n) del p(re)se(n)te mo(n)do et come i(n) de lla [morte] è di(s)p(re)siato da tuta çente.

F (29r): De lli loldi et nobilitade de lla n(ost)ra do(n)na e s(ant) a mare di Dio p(er) li qualli magnificamente è exaltada e quanti beni li pecatori e lli giusti p(er) llei ricieveno e riceverano mo'e senpre.

G (33r): Oracio(n) devotisima e[di]fica[n]te inprima a lla Mado(n)na, dapoi al Fiuol di Dio, e dapoi al Padre e poi a llo Spirito S(ant)o, ultimame(n)te a tucta la Ternitade.

Come si può facilmente constatare, $\mathrm{i}$ titoli dei primi due componimenti rappresentano una traduzione letterale di quelli registrati in V. È verosimile che nell'archetipo di $\mathrm{S}$ e $\mathrm{V}$ anche i testi restanti fossero corredati da rubriche, tutte in latino, andate perse nel corso della tradizione manoscritta (probabilmente a causa di una dimenticanza di qualche copista, visto che i titoli si trascrivevano spesso dopo il testo principale e non contemporaneamente ad esso) e conservate in S sotto forma di una traduzione (a un volgarizzamento fanno pensare anche alcuni tratti latineggianti che presentano le rubriche di $\mathrm{S}^{8}$, di cui verrà detto sotto) ${ }^{9}$. S presenta anche qualche titolo 'secondario' (rubriche che si trovano all'interno dei componimenti), in latino dopo il v. C60 (12r): Incipit de amore Christi e alternativamente in volgare e in latino nel testo G,

8 Tale tendenza è naturale, visto che si tratta - "pur nella visione sincronica che il Medioevo ha dei rapporti tra latino e volgare, in quello che potrebbe definirsi un bilinguismo e biculturalismo in senso sincronico" - di "un tradurre "verticale', dove la lingua di partenza, di massima il latino, ha un prestigio e un valore trascendente rispetto a quella d'arrivo [...], è un modello ideale o addirittura uno stampo nel quale si versa per ricevere forma il materiale di fusione", contrapposto a "un tradurre 'orizzontale', o infralinguistico, fra lingue di struttura simile e di forte affinità culturale come le romanze" (Folena 1991: 13). Tuttavia, il modello latino non condiziona mai in modo 'meccanico' il volgarizzamento, ma va considerato piuttosto come "acceleratore e regolarizzatore dell'attività creativa e innovativa del volgare" (Segre 1953: 20).

9 Perché sono state volte in un volgare settentrionale queste rubriche? Ricordando che si tratta di componimenti - e anche di un manoscritto - di matrice francescana, e per di più di testi omiletici, ci sembra pertinente adottare la spiegazione che adduce Carlo Delcorno per i volgarizzamenti religiosi tardomedievali in generale: "I volgarizzamenti religiosi esorbitano [...] da motivazioni puramente letterarie o dal gioco delle leggi del mercato librario, e sono piuttosto da studiare come riflesso della duplice fondamentale funzione religiosa del linguaggio: la predicazione, anche nella forma della muta praedicatio affidata al libro di meditazione, e la traduzione dei testi sacri, che ingloba tutto quanto serva a commentare, dilatare, farcire e illustrare la Bibbia" (Delcorno 1998: 6); in sintesi, l'interesse e il valore di questi documenti consiste non solo e spesso non tanto nelle loro caratteristiche interne, ma soprattutto nella possibilità di risalire, indagando la tradizione manoscritta di questi testi, a delle informazioni sul loro pubblico. Il fatto stesso di adottare uno o altro codice linguistico era significativo: i libri in latino erano rivolti al pubblico dotto di chierici e universitari, mentre le traduzioni dal latino e dal francese - la cui produzione raggiunge il suo culmine fra Due e Trecento - erano indirizzati ai laici oppure a ordini religiosi femminili e terzi ordini maschili organizzati nelle confraternite (De Luca 1963: 16). 
per introdurre ogni nuova preghiera: dopo il v. G60 Oracionne della nostra Donna (in volgare), dopo il v. G110 L'oracion del Fiuol de Dio (in volgare), dopo il v. 174 Horaçio a patrem (in latino, con $<\mathrm{h}>$ iniziale aggiunta per ipercorrettismo), dopo il $\mathrm{v}$. G309 Oracio ad spiritom sanctom $<m>10$ (in latino, ma l'errore nella desinenza - om per $u m$ - fa pensare a un passaggio verso le forme volgari: spirito $(m)$ sanctom $<m>$ probabilmente deriva da spirito santo con la desinenza volgare -o), dopo il v. G328 Oracio a matrem (in latino), dopo il v. G344 Oracio a Ternitatem (in latino, ma con la metatesi del volgare), dopo il v. G364 Oracio ad Sanctam Mariam (in latino), dopo il v. G396 Oracion comune cossi per li vivi come per li morti (in volgare). In V tutti questi titoli sono assenti, ma al loro posto si riscontrano degli spazi bianchi tra le righe di testo, esattamente prima dei versi che in S sono preceduti dalle rubriche riportate sopra, cosa che fa ipotizzare la presenza di tutti questi titoli - in latino - già nell'archetipo di $\mathrm{V}$ e S.

I titoli in volgare non possono essere ricondotti all'archetipo veronese, visto che manca qualsiasi traccia di veronesismi fonetici, presenti invece, pur sporadicamente, nel testo dei componimenti tràdito da $\mathrm{S}$ (inoltre è poco probabile che $\mathrm{i}$ titoli in latino presenti in $\mathrm{V}$, siano una traduzione delle rubriche redatte originariamente in volgare). D'altra parte, i titoli non possono esser stati volgarizzati dal copista dello stesso codice $\mathrm{S}$, il che si evince dai seguenti errori (attribuibili al trascrittore di $\mathrm{S}$ o già presenti nel suo antigrafo): e ficate $\mathrm{G}$ per edificante, l'omissione di un sintagma nominale dopo la preposizione articolata in de lla nella rubrica $\mathrm{E}$ (abbiamo integrato per congettura morte, basandoci sul contenuto del componimento), nonché - anche se è meno cogente - dalla mancata concordanza del participio con il sostantivo in opra ch'avrà fate $\mathrm{C}$ (corretto da noi in opra ch'avrà fata), errori che dimostrano che si tratta sicuramente di una copia. Il volgarizzamento deve aver avuto luogo a uno stadio intermedio della tradizione e risale verosimilmente al trascrittore di una delle copie settentrionali piuttosto tarde.

\section{Il dato materiale}

Prima di esaminare i tratti linguistici che presentano le rubriche di S, forniamo una breve descrizione delle loro caratteristiche paleografico-codicologiche. I titoli sono trascritti all'interno dello specchio di scrittura, dal punto di vista della mise en page l'unica differenza tra le rubriche e il testo del componimento consiste nel fatto che i titoli, essendo in prosa, occupano tutto lo spazio dello specchio di scrittura. Le rubriche sono eseguite ad inchiostro rosso e appartengono tutte quante alla stessa mano, la quale non sembra diversa da quella che ha copiato i componimenti. I titoli sono stati aggiunti dopo la trascrizione del testo principale, cosa che si vede molto

10 Le parentesi uncinate indicano le espunzioni. 
bene dalla disposizione del testo alla pagina 23v: la seconda riga della rubrica oltrepassa i limiti dello specchio di scrittura, ma nonostante questo lo spazio non basta e il testo arriva ad occupare anche una parte della riga successiva, dopo il primo verso del componimento E.

\section{Tratti linguistici}

Se la versione dei testi di S fa trasparire un ascendente redatto in antico veronese (nonostante i veronesismi del codice colombino abbiano una distribuzione sporadica, in quanto residui di uno strato antico, nella maggior parte dei casi cancellato o modificato), le rubriche del nostro manoscritto non presentano tratti caratteristici del veronese antico - riscontrabili anche in $\mathrm{V}$ e spesso anche in $\mathrm{U}$ e $\mathrm{O}$. I tratti 'veronesi' registrati nel testo principale e assenti nelle rubriche sono i seguenti: la metafonesi da $-i$, che coinvolge $e \mathrm{e}$ $o$ toniche ${ }^{11}$, l'esito $\mathrm{AU}>o n^{12}$, l'apocope dopo dentale ${ }^{13}$ e l'apocope sillabica ${ }^{14}$, la vocale d'appoggio $-o$, reintegrata in seguito alla caduta della $-e$ etimologica ${ }^{15}$, la sincope in

11 S presenta la metafonesi di $\rho$ in rasuri B95 'rasoi' e russi A257 'rossi' (le forme che avevano anche V e U e che rappresentano tracce dell' archetipo veronese) e probabilmente anche in loguri B93 'lucertoloni, ramarri' (V ligori, U liguri), se si postula il singolare logoro e non loguro (quasi sicuramente da correggere in legoro / leguro: sulle forme di questa parola nei volgari settentrionali cfr. Mussafia 1864: 223, TLIO: s. v. liguro).

La metafonesi di e si osserva in S quilli E300, E485 e quigli A72 'quelli' (gli altri tre codici presentano forme metafonetiche: V quigi, U quili, quilli, O qui'). Sulla metafonesi veronese cfr. Riva 1953: 9; Corti 1960: 49; Cornagliotti 1979: 210; Verlato 2002: 16; Bertoletti 2005: 46-47, ecc. Per ragioni di leggibilità, nella descrizione di fenomeni linguistici non si segnala invece lo scioglimento di abbreviazioni.

12 S presenta cons(s)a A168, E75, F153, F159, G252, G350 'cosa' accanto a cossa A71 e passim e cosse A40 e passim. Su questo fenomeno negli antichi testi veronesi cfr. Cornagliotti 1979: 210; Verlato 2002: 17; Bertoletti 2005: 61-64, ecc.

Le parentesi tonde negli esempi tratti dal testo dei componimenti qui e in seguito segnalano che nel manoscritto sono attestate sia consa che conssa, sia beleça che belleça, ecc.

13 S presenta l'apocope dopo dentale in serpent A54 e çent A53 'gente', di fianco a tanti esempi che presentano il mantenimento o la restituzione della vocale finale: ç(i)ente A55 e passim e giente A72 e passim 'gente' (V e U avevano çent o çento, $\mathrm{O}$ reintegra: çente, cento o gente; quanto all'altro vocabolo, al v. A54 tutta la tradizione manoscritta presenta la forma apocopata serpent), ecc. Sull'apocope veronese cfr. Riva 1951: 26-28; Corti 1960: 49; Caprettini 1971; Bertoletti 2005: 116-121, ecc.

14 S presenta le forme apocopate me 'A218, D276, D293, G160 'mio' accanto a meo B26, D41, G48 e mio B73 e passim; De'A220, G159, G254 'Dio' accanto a Deo A236 e passim e Dio A6 e passim, ecc.

15 S presenta questo fenomeno negli infiniti: esro A56 'essere' accanto a es(s)ere A76 e passim ed eser B226, D45 (V sempre esro, O sempre eser, U alterna esro ed eser); metro B131 e metero B82 'mettere' accanto a metere C188 e meter A184, B131 (V sempre metro contro U meter), ecc.; nelle forme della terza persona singolare del presente indicativo: rendo C63, C144 'rende' accanto a rende A92 e passim (V sempre rendo, $\mathrm{O}$ sempre rende, $\mathrm{U}$ alterna rendo e rende), ecc.; nei sostantivi femminili della terza declinazione: parto A22, A31, A33 'parte' accanto a parte A222 e passim (V sempre parto contro 
postonia $^{16}$, il passaggio -LLI > $-g i^{17}$. Come è logico aspettarsi, nelle rubriche si osservano molti dei fenomeni dai quali è caratterizzata la lingua del codice $S$; tuttavia, dal punto di vista linguistico i titoli rappresentano un quadro un po' diverso da quello costituito dai tratti riscontrati nel testo dei componimenti. I fenomeni di rilievo registrati nelle rubriche sono descritti qui sotto, in confronto con il materiale del resto del codice; uno spazio minore è riservato ai tratti aspecifici.

\subsection{Grafia}

\subsection{1. $<$ l $>$ e $<$ s $>$ geminate}

Vista la provenienza settentrionale del codice, è fondato interpretare le consonanti doppie come un fatto grafico e non fonetico (è noto che lo scempiamento delle geminate è un tratto fonetico pansettentrionale). In S sono frequenti i raddoppiamenti consonantici che non si spiegano con processi di fonetica storica e vanno considerati come fenomeni puramente grafici; è un tratto attestato frequentemente nei testi di provenienza settentrionale, soprattutto veneti ed emiliani (cfr. l'ipotesi riguardante le grafie raddoppiate per le consonanti dal tratto 'lungo' in Stussi 1965: XXX, nonché Borgogno 1980: 55); in V, U e O i raddoppiamenti di questo tipo sono quasi del tutto assenti. S presenta le grafie come candella F184 e candelle E177 e passim; malla A25 e passim 'cattiva' (per la <-11->) oppure ascossa C82 e passim 'nascosta' accanto a ascoso E242 'nascosto'; bossia A165 'bugia' accanto a bosia E319, ecc. $($ per la $<-$ ss- $>$ ).

Il codice $\mathrm{S}$ presenta spesso anche delle doppie etimologiche (ciò non riguarda solo $<$-ll- $>$ e $<$-ss- $>$, ma anche altre consonanti), in contrapposizione agli altri tre codici che presentano di norma una consonante scempia, come attestano le coppie

U parte e O parte, part), ecc.; negli indeclinabili: senpro C280 'sempre' accanto a s(i)enpre A82 e passim (V sempre sempro contro OU senpre). Si tratta di uno dei fenomeni più caratteristici dell'antico veronese, cfr. sull'argomento Riva 1953: 24-25; Corti 1960: 48; Rohlfs 1966-69: § 143; Cornagliotti 1979: 208-209; Verlato 2002: 16-17 e Verlato 2009: 364-366; Bertoletti 2005: 123-127, ecc.

$16 \mathrm{~S}$ presenta la sincope in questa posizione soprattutto negli infiniti: desbatro D83 'muoversi in modo disordinato e scomposto; agitarsi' di fianco alla forma con il reintegro della $e$ postonica interna (ed -e finale) desbatere B310 (in V e U si aveva desbatro in entrambi i casi), esro A55 'essere' accanto a essere C150 e passim ed eser B226, D45 (V sempre esro, O sempre eser, U esro ed eser), ecc. Sul fenomeno cfr. Riva 1953: 24; Cornagliotti 1979: 209; Verlato 2002: 19; Bertoletti 2005: 101-116.

17 In S la palatalizzazione si riscontra in restegi B181 'rastrelli' (V rastegi, U rostigi) e probabilmente anche in quigli A72 'quelli' (se la grafia $<\mathrm{gl}>$ in questo caso ha valore di affricata palatale sonora [d3], come avviene talvolta in testi veneti). L'esito palatale in questa posizione è un fenomeno che contrappone Venezia a tutta la terraferma veneta, cfr. Riva 1953: 34; Ineichen 1966, II: 378-379; Rohlfs 1966-69: $\S$ 233; Pellegrini 1977: 63; Verlato 2002: 18; Tomasin 2004: 150-151; Bertoletti 2005: 180-182, ecc. 
di varianti quali S beffe] UV befe A166 (ma O beffe); S donna] OUV dona A249 e passim; S Madonna] V Madona F56 e passim; S roccha] V rocha F198; S appella] V apella F131; S falla] V fala F94 'sbaglia'; S glossa] V glosa F199, ecc. In tutti questi esempi S conserva, almeno graficamente, la consonante doppia dell'etimo latino e del corrispondente toscano.

Nelle rubriche si osserva un uso delle consonanti scempie e doppie coerente con la lingua del codice $\mathrm{S}$. Il raddoppiamento è etimologico in belleçça $\mathrm{A}$, allegreçe $\mathrm{A}$, quella $\mathrm{AB}$; non è etimologico invece in Babillonia $\mathrm{B}$, qualli $\mathrm{F}$, lli $\mathrm{F}$, llei $\mathrm{F}$. È pressoché regolare nelle preposizioni articolate, che presentano le forme sintetiche allo $\mathrm{G}$, alla $\mathrm{G}$, dell' $\mathrm{C}$, della $\mathrm{ABCEF}$, delli $\mathrm{F}$, nonostante si riscontrino anche le forme analitiche de l'ultimo $\mathrm{D}$, de l'omo $\mathrm{E}$.

D'altra parte, la rubrica del componimento $\mathrm{G}$ presenta devotisima, una grafia con lo scempiamento della sibilante geminata latina, un altro esempio dell'impiego sporadico non etimologico delle consonanti semplici e doppie.

\subsubsection{Raddoppiamento dell'affricata dentale sorda $<$ ç>}

È attestato in belleçça A, e probabilmente in questo caso il raddoppiamento grafico si spiega con la tendenza ad aderire al modello toscano e quindi deriva da una reale pronuncia geminata. Nel testo del manoscritto il fenomeno si riscontra solo in façça F179, G13, G43 'faccia', altrimenti si ha bel(l)eça A144 e passim, bel(l)eçe A31 e passim, ecc.: in questi casi la scelta di una consonante semplice sembra essere dovuta alla forma dell'antigrafo, riprodotta fedelmente (mentre nella rubrica A si trattava di tradurre pulcritudine).

\subsection{3. $<\mathrm{i}>$ diacritica}

$\grave{\mathrm{E}}$ attestata in ricieverà $\mathrm{D}$, ricievono $\mathrm{F}$, ricieverano $\mathrm{F}$ (davanti a vocale palatale) e in ciascun $\mathrm{D}$, giudicio $\mathrm{D}$, giusti $\mathrm{F}$ (davanti a vocale velare).

L'inserimento della $<\mathrm{i}>$ diacritica in sillaba tonica è un fenomeno ampiamente attestato in $\mathrm{S}$ e assente in $\mathrm{V}, \mathrm{U}$ e $\mathrm{O}$. Si registra dopo le seguenti lettere:

\footnotetext{
$<\mathrm{c}>$ (davanti sia a vocale palatale che velare): ciel(lo) A14 e passim; ciercha A41 'vicino' accanto a cercha B208; prociede E77 'proviene'; reciever D201 'ricevere', ecc.; ciascun A45 e passim, ecc.

$<c ̧>$ (davanti sia a vocale palatale che velare): çiemo D36 'gemo'; çiente C112 e passim 'gente'; ançiolo B331 e ançiol(l)i A239 e passim 'angelo, angeli'
} 
accanto a ançolo A235 e ançoli A122, D230; asaçia C279 'assaggia', çioe C119 'gioie' accanto a çoie C172, ecc.

$<\mathrm{g}>^{18}$ (davanti sia a vocale palatale che velare): argiento A58 e passim; angielic(h)a C36 e passim e angieliche F29; giemma C81 e passim, ecc.; vegiando D21 'vedendo'; Giovani A32 e Giovane B271; giudisio D17, ecc.

$<\mathrm{x}>\mathrm{e}<\mathrm{s}>$ (davanti sia a vocale palatale che velare): baxia G162 'bacia' e baxiasi F154 'baciasti'; bruxia B283 'brucia' contro bruxante D242 'bruciante, che causa un intenso dolore'; paxie $\mathrm{C} 188$ e passim, pas(s)ie $\mathrm{C} 16$ e passim 'pace' accanto a paxe G183, G198, G205, ecc.

$<$ gn $>$ (davanti a vocale velare): benignia G403; cogniosca G504, ecc.

Questa aggiunta di una vocale diacritica in contesti fonetici dove la sua presenza non è richiesta (soprattutto dopo $<$ ç $>$ con valore di affricata palatale sonora, $<$ s $>$ e $<x>$ con valore di sibilante dentale sorda, e davanti a vocale velare), sembra un incrocio tra la grafia settentrionale e il modello toscano (Raugei 1984: 27; Gambino 1996: 236; Tomasoni 1973: 165).

La peculiarità delle rubriche consiste nel presentare la $<\mathrm{i}>$ diacritica dopo $<\mathrm{c}>$ anche in sillaba atona (ricieverà, ricieverano), cosa che non si osserva nel testo dei componimenti; infatti, si ha sempre celeste Atit e passim; celestial A279 e passim, ecc; dello stesso verbo ricevere sono attestate le forme reçever $\mathrm{C} 195$ 'ricevere', receve F218 'riceve', recevesti G144 'ricevesti', recevuda G343 'ricevuta', recevando $\mathrm{C} 255$ 'ricevendo', tutte senza la $<\mathrm{i}>$ diacritica.

\subsubsection{Grafie latineggianti}

La grafia $<\mathrm{ci}>$, usata per rendere il nesso affricata dentale sorda + iod, è etimologica in giudicio $\mathrm{D}$ e semidotta in operacion $\mathrm{C}$, oracion $\mathrm{G}^{19}$; il suo impiego è coerente con l'usus scribendi di S: cfr. tentacion A263 contro V tentation, U temptation, $\mathrm{O}$ t[ent]ation; aflicion D76 contro V afliction; consiencia C213 contro V conscientia; nacion $\mathrm{F} 72$ contro $\mathrm{V}$ nation, ecc.

Anche la grafia ipercorretta $<\mathrm{ct}>$ per $<\mathrm{t}>$, riscontrata in bructeça $\mathrm{B}$, tucta $\mathrm{G}$, si inscrive nella tendenza generale dell'estensione pseudoetimologica dei nessi

18 I primi tre grafemi $(<\mathrm{c}>,<\mathrm{c}>\mathrm{e}<\mathrm{g}>)$ sono allografi e rappresentano l'affricata dentale sonora [dz], cfr. le grafie centil G45, cientil G402, çentil C19, çientil A225, gientil A133, A249 e gientille C56 'nobile, eccelente, perfetto'. I grafemi $<\mathrm{c}>\mathrm{e}<\mathrm{c}>$ possono essere impiegati anche per l'affricata palatale sorda [ts], cfr. ciel(lo) A14 e passim e çiello B332, G286.

19 Queste abitudini grafiche delle scriptae volgari erano influenzate dal fatto che anche nel latino medievale ci fosse una variazione libera nell'impiego delle grafie $<\mathrm{ci}>\mathrm{e}<\mathrm{ti}>$. 
$<\mathrm{ct}>\mathrm{e}<\mathrm{pt}>$ per $t$, attestata in S: lecticia $\mathrm{F} 70$ contro V leticia; profecta $\mathrm{C} 322 \mathrm{e}$ profecti A125, F189 contro V profeta, propheti, O profeti (U presenta una forma compendiata); tucto E189, tucta A41 e passim; tucti A122 e passim, tucte A59 e passim, tuctore A234 contro OUV tuto, tuta, tuti, tute, tutore; destrepto B254 'intransigente, austero, severo' contro UV destreto; dripta C58 'destra' contro V drita, ecc.

Il segno $<\mathrm{x}>$ per la sibilante dentale sonora viene usato solo in exaltada $\mathrm{F}$ 'elevata alla grazia divina', di cui è probabilmente responsabile l'influsso della grafia latina, mentre altrove in $\mathrm{S}$ questo grafo è frequentemente usato per rappresentare tale consonante anche in contesti non etimologici (gli altri tre codici impiegano $<\mathrm{x}>$ più raramente): Jeruxalem A5, G341 (V Jerusalem, U Jeruxalem, O Geruxalem), ma Jerusalen nella rubrica A; luxe A78 'splende' accanto a luse A192 (UV luso contro O luxe); roxa A194, F9, ruoxa F33 e ruoxe C175, C297, D365 accanto a rosa A252, C237, ruosa A134, A228, C233 e ruose A92 (UV rosa, rose, O rosa accanto a roxe), ecc.

Aspecifico è l'uso di $<\mathrm{j}>$ nel latinismo Jerusalen A. Nella pronuncia reale ci doveva essere un'affricata sonora dentale; anche al v. A5 si ha Jeruxalem (anche V e U presentavano questa grafia latineggiante, in $\mathrm{O}$ si ha Gerusalem, grafia semidotta o toscaneggiante).

\subsection{Fonetica}

3.2.1. Il codice $\mathrm{S}$, a differenza di $\mathrm{V}, \mathrm{U}$ e $\mathrm{O}$, è caratterizzato da un abbondante dittongamento spontaneo di tipo padovano-veneziano. Nelle rubriche, tuttavia, il dittongo di ŏ si riscontra solo in fiuol G 'figlio' (così S presenta fiuol A135 e passim). Invece in core $\mathrm{C}$ il dittongo manca, mentre nel testo principale la forma con dittongo cuor A183 e passim prevale su quella con monottongo cor A246 e passim; è attestata anche la forma cur $\mathrm{C} 271$, con la riduzione del dittongo tipica del padovano antico (Ineichen 1966, II: 357; Tomasin 2004: 105-106, ecc). La mancanza del dittongo in core si spiega probabilmente con il fatto che il volgarizzatore avesse di fronte il testo latino.

\subsubsection{Esito $\mathrm{AU}>\boldsymbol{o l}$}

La velarizzazione $\mathrm{AU}>$ ol davanti a consonante dentale in $\mathrm{S}$ è attestata in posizione atona in goltade D237 'schiaffi' < gall. GAUTA (V galtae); (h)oldir A1 e passim 'udire, sentire' e in posizione tonica in golça E233 'goda' < GAUDEAT (V aveva la stessa forma) e smeroldi A213 accanto a smeraldi A260 (V smeraldi, OU 
smeraldo), forma per la quale si presuppone uno stadio intermedio con au (DELI: s. v. smeraldo). $\mathrm{V}, \mathrm{U}$ e $\mathrm{O}$ presentano l'esito $\mathrm{AU}>o l$ solo in posizione atona, come in oldir A1 e passim. La rubrica del componimento F presenta questo esito in posizione tonica in loldi 'lodi', mentre nel testo dello stesso componimento il vocabolo in questione si registra nella forma latinizzata laudi F133, con il mantenimento del dittongo latino; lo stesso vale anche per le voci del verbo lodare: laudo F55 'lodo' e lauda B72, F57 (2 vv.) 'loda', si conserva au anche in posizione atona in laudar A154 e passim (il resto della tradizione concorda in questo con $\mathrm{S}$, a eccezione di $\mathrm{O}$, che presenta l'esito semidotto del dittongo in laodare A120, laodando A131 accanto a laudar A154 e laudando A135 e passim). L'esito ol è tipicamente veneziano; tuttavia, è compatibile anche con la fonetica di altri volgari settentrionali (Contini 1938: 311; Ghinassi 1965: 92; Rohlfs 1966-69: $\S \S 17,42$; Arcangeli 1990: 7-9, ecc.); la scheda del corpus testuale dell'Opera del Vocabolario Italiano $(\mathrm{OVI})^{20}$ riporta attestazioni di forme del sostantivo loldo o lolde (è maschile, cfr. 3.3.2) e del verbo loldar in testi veneti, tra cui la veneziana Legenda de Santo Stadi (Badas 2009: 110 e passim), il volgarizzamento veneziano dei Vangeli (Gambino 2007: 15 e passim), ecc., e in testi emiliani, tra cui le dicerie in volgare bolognese (Frati 1911: 331 e 333), il laudario dei Battuti di Modena (Elsheikh 2001: 4 e passim), ecc.

\subsubsection{Esito di $\check{E}$ in iato}

Le rubriche D e F presentano la forma Dio con l'esito $\breve{\mathrm{E}}>i$. Questo esito è frequente in S (mentre V, U e O conservano e): Dio A6 e passim, accanto a Deo D41, De' E236, F50, G159 ecc. e Dee A220 (V Deo, De', OU Deo); dia E328, G461 (V dea); io $\mathrm{A} 33$ e passim accanto a $e^{\prime} \mathrm{F} 62$ e eo passim (UV eo, e', $\mathrm{O} e$ '); rio $\mathrm{B} 164$ e passim (UV reo, re'); ria B164 e passim (UV rea); rii B201, D275 (UV rei); stia B170 (UV stea), ecc. L'esito $i$ è veneziano o toscaneggiante; tuttavia, entrambi gli esiti sono attestati nei testi veneti, senza una distribuzione netta ed uniforme (Bertoletti 2005: 56 , con altra bibliografia).

\subsubsection{Esito E $>i$}

I titoli presentano i prefissi ri- e non re- in ricievono $\mathrm{F}$, ricieverà $\mathrm{D}$, ricieverano $\mathrm{F}$, dis- e non des- in dispresiato $\mathrm{E}$ 'disprezzato', in e non en come preposizione (CDE) e come prefisso (inprima G 'all'inizio'), nonché l'esito di (A, B, D) della preposizione $\mathrm{DE}$ accanto a de $\mathrm{C}$ e de $\mathrm{A} 8$ e passim nel testo dei componimenti. La

20 Tutti i riscontri tra il nostro manoscritto e altri testi che abbiamo riportato nel presente articolo sono stati individuati grazie all'interrogazione di questo strumento, consultabile all'indirizzo http:// gattoweb.ovi.cnr.it/. 
chiusura $\mathrm{E}>i$ nei prefissi e nelle preposizioni de e en è un toscanismo frequente in veneziano e in padovano antico (Rohlfs 1966-69: $\S 130$; Bertoletti 2005: 82-83, con altra bibliografia). S presenta l'esito $i$ abbastanza spesso, ma nel testo principale, a differenza delle rubriche, non mancano esempi della conservazione della vocale etimologica: rimaner D162 accanto a remaner D322; risplendente F204 accanto a resplende $\mathrm{A} 80, \mathrm{C} 40, \mathrm{D} 388$, resplendente $\mathrm{A} 144$ e respiandente $\mathrm{F} 51$; lo stesso verbo ricevere presenta le forme reçever $\mathrm{C} 195$ 'ricevere', receve F218 'riceve', recevesti G144 'ricevesti', recevuda G343 'ricevuta', recevando C255 'ricevendo'. V, U e O conservano di norma la vocale $e$.

\subsubsection{Passaggio $\mathrm{U}>\boldsymbol{o}$ in protonia}

Il fenomeno si osserva in ponidi B 'puniti' ed è coerente con il quadro generale che presenta S: anonciando G301 'annunciando'; circondado A90 e circondade B39 'circondato, circondate'; enfondada A44 'fondata'; osure B300 'usure'; ponçente D244 'pungenti', ecc. V presenta spesso in questi contesti la vocale $u$ : annunciando, circundao e circundae, fundaa, ecc., e così $\mathrm{O}$ e U: $\mathrm{O}$ circundà, U circundado e circundae, ma OU fondà; il mantenimento della $u$ sembra un fenomeno puramente grafico (latinismo); l'innovazione di S consiste piuttosto nell'avvicinare, in questo caso, la grafia alla pronuncia effettiva.

L'assenza del passaggio $\mathrm{U}>o$ nella parola suave della rubrica $\mathrm{C}$, si dovrà spiegare sempre per latinismo (la rubrica latina che servì da punto di partenza per il nostro titolo in volgare doveva contenere suavis); nel testo principale leggiamo sempre soave $\mathrm{C} 45, \mathrm{C} 133, \mathrm{C} 261, \mathrm{C} 263$ (si tratta dello stesso componimento a cui è riferito il titolo che contiene suave).

\subsubsection{Apocope}

La mancanza dell'apocope dopo dentale (presente $\mathrm{D}$, çente $\mathrm{E}$ 'gente', conforto $\mathrm{D}$, nasimento E 'nascita') contrappone le rubriche all'archetipo veronese (cfr. sopra nota 13$)$.

Il testimone veronese (V) è caratterizzato da un'apocope molto avanzata, sporadica dopo dentale e quasi regolare dopo nasale e liquida, una situazione simile si osserva anche in $U$, anche se l'apocope coinvolge in questo codice meno contesti fonetici (ad esempio, la - $i$ finale dopo liquida è conservata); $\mathrm{O}$ si avvicina a $\mathrm{S}$ nel restituire le atone finali. Il testo principale di S presenta un quadro piuttosto disordinato, in seguito alla sovrapposizione di strati diversi corrispondenti a diversi stadi della tradizione manoscritta: 
-e dopo liquida: amore C63, F4 accanto a amor A189 e passim (OUV amor); creatore A222, G118 e criatore F176 accanto a creator A24, A120, A147 e criator B243, G175 (UV sempre creator, O alterna creator e creatore); dolore C31, F149, G117 accanto a dolor B323 e passim (UV sempre dolor); melle C66 e mielle C99 'miele' accanto a mel A104, D286 (V sempre mel contro U mele), ecc.

$-i$ dopo liquida (nei plurali): martori A133 'martiri' (V martir contro U martiri e O martori); pecadori D146, D340 e pecatori B155, D211, D278, F168, accanto a pecador D220, D291 (V peccaor contro U peccatori); sospiri D5, E93 (V sospir), ecc.

-e dopo nasale: cane B108 (V can contro U cane); nome A9 e passim (V nomo, nom, $\mathrm{U}$ nome, nomo, $\mathrm{O}$ nome); pasione $\mathrm{C} 195$ accanto a passion $\mathrm{G} 150$ (V passion); tençone D9 (V tençon), ecc.

-o dopo nasale: Adamo F187 (V Adam); bono C247 accanto a bon A12 e passim (OUV bon), ecc.

Come si può facilmente osservare, $\mathrm{S}$ ripristina frequentemente le vocali finali cadute in V; tuttavia, questo fenomeno è piuttosto fluttuante e il codice oscilla tra le forme apocopate e quelle piene. Quanto alle rubriche, le forme attestate sono poche per parlare con certezza di una regola; tuttavia, i fenomeni riscontrati formano un quadro ordinato che non conosce eccezioni: si osserva l'apocope di -e e -o dopo nasale in ciascun $\mathrm{D}$, operacion $\mathrm{C}$, oracion $\mathrm{G}$, in concomitanza con il mantenimento della $-e$ e della $-i$ dopo liquida: amore $\mathrm{C}$; core $\mathrm{C}$; pecatori $\mathrm{BF}$. La situazione presente nelle rubriche è compatibile con il vocalismo padovano (Ineichen 1966, II: 364365; Pellegrini 1977: 63), mentre in tutto il resto del Veneto si registra la caduta della vocale finale anche dopo liquida (per la situazione veneziana cfr. Stussi 1965: XXXIII; Sattin 1986: 72-73; Gambino 2007: LXXVI-XXVII, ecc.); la restituzione delle vocali finali dopo liquida è anche bolognese (Corti 1962: LIII; Raugei 1984: 45; Andreose 2002: 659, ecc.). Non si può comunque escludere che la restituzione delle - $e$ finali sia dovuta, come una serie di altri fenomeni, al fatto di essere condizionati dal testo latino che si traduce.

\subsubsection{Epitesi di -o nelle forme verbali}

Nelle forme verbali della terza persona del plurale sono $\mathrm{B}$, ricievono $\mathrm{F}$ e ricieverano $\mathrm{F}$, formate alla toscana (le forme della terza persona plurale con desinenze proprie sono un elemento estraneo al sistema linguistico veneto e vanno ascritte all'influsso toscano, cfr. anche 3.3.4), si riscontra l'epitesi di -o, anch'essa un toscanismo (stavolta fonetico). 


\subsubsection{Esito sonoro della consonante occlusiva dentale sorda intervocalica $t$}

La lenizione dell'occlusiva sorda dentale intervocalica è attestata in exaltada F 'elevata alla grazia divina', pecadi $\mathrm{B}$ 'peccati', ponidi $\mathrm{B}$ 'puniti', nobilitade $\mathrm{F}$ 'nobiltà' (forma del femminile plurale), ternitade $\mathrm{G}$ 'trinità' (forma del femminile singolare). Nel presentare questo tratto le rubriche di S si discostano dall'archetipo (in $\mathrm{V}$ si ha quasi sempre il dileguo della consonante intervocalica, e questo esito rende possibile delle rime perfette come amalai A103 'malati': mai A104; albergaria A279 'accoglienza' : complia A280 'compiuta, finita'; le rime di questo tipo ci autorizzano ad ascrivere questo tratto anche alla lingua dell'originale) e concordano con il resto della redazione $\mathrm{S}$, che nella stragrande maggioranza dei casi presenta la dentale intervocalica nella forma sonora: aidar B272, D395 'aiutare', aidadi G382 'aiutate' e aida G27, G80 ‘aiuto’ accanto ad aitar B268 (V aveva aiar, aiai, aia, U aiar); abiadi A87, B333 'abbiate' (V abià, abiai, U abiai, O abià); abitador A76 'abitante' (OUV abitaor); afadiga B252 'affatica' (V afaiga, U faiga); faciadi F232 'facciate' (V façai); recordador C129 'colui che ricorda' (V recordaor); sede B308, D217 'sete' accanto a seo A88 (V seo e O se' contro U sede); tapedi F209 'tappeti' (V tapei); voiadi 'vogliate' A20 (UV voiai, O voià $<r>$ ), ecc. Come si può constatare, la sonorizzazione è sistematica, al punto tale da incidere sulla morfologia (così nasce la desinenza verbale della seconda persona del plurale -adi) e sulla formazione delle parole (così il suffisso -dor, come in recordador). La desinenza -adi non si registra mai in $\mathrm{U}$, nonostante anche questo codice presenti talvolta la $-d$ - intervocalica derivante da -T-; oltre alla forma sede, riportata sopra, possiamo citare per U corrador A49 'corridoi' (SV corraor, O coreori), arsirado A55 'paralitico, sciancato’ (S arsirado, V asirao, O asirà), ecc. Qualche volta l'esito sonoro è presente in vocaboli mancanti nei rispettivi versi di V e comparsi in S per errore: afamadi B96 'affamati', seda F212 'seta'. Tale - $d$ - intervocalica sembra una restituzione tarda veneziana o venezianeggiante, un fenomeno che si diffonde sempre di più nella seconda metà del Trecento (Ascoli 1873: 458; Corti 1966: 136; Pellegrini 1966: 100; Sattin 1986: 80-81; Arcangeli 1990: 13-14; Burgio 1995: 47; Ferguson 2005: 497 e 499; Gambino 2007: LXXIII, ecc.); tuttavia, non si può neanche escludere la provenienza emiliana di questo fenomeno (Corti 1962: LIV; Raugei 1984: 46-47; Elsheikh 2001: XL, ecc.).

L'esito -ade nei sostantivi in -ATE(M) è caratteristico, anche se non esclusivo, della scripta veneziana (Ferguson 2005: 496 e 498); tale esito è assente in V e frequente in S: benignitade G147 accanto a benignità G360 (V sempre benignità); fraçilitade E4 'fragilità' accanto a fragilità E30 (V fragilità); istade D334 'estate' accanto a istà B199, D99 (V istao D334, istà B199, D99); pietade G5 e passim accanto a pietà C11 e passim (V pietà); segurtade G126 'sicurezza' accanto a segurtà A197 (OUV segurtà) e ternitade G329 'trinità' accanto a ternità A154, G294, G332, G345 (UV 
trinità, O ternità). Al plurale si osservano unicamente forme integre: bontade C94, F44, F177 (V bontae C94, F44 e bonté21 F177); podestade D134 (V poesteae).

L'esito sordo in beatitudine A si spiega per latinismo, tanto più che nella rubrica latina di $\mathrm{V}$ si ha la stessa forma (beatitudine). La sorda di pecatori $\mathrm{BF}$ e di dispresiato E ‘disprezzato' sembra essere dovuta sempre a influssi dotti, toscani o latini.

L'esito - $d r$ - del nesso -TR- in padre $\mathrm{G}$-contro il dileguo della dentale in mare $\mathrm{F}$ 'madre'- non è veronese (potrebbe essere un tratto 'normalizzante' del veneziano o dell'emiliano, come la restituzione della dentale intervocalica di cui sopra); tuttavia, è molto probabile che la forma in questione sia semplicemente un toscanismo apportato da un copista di provenienza settentrionale.

\subsubsection{Trattamento dell'occlusiva bilabiale sorda intervocalica}

Si registra l'assenza della lenizione della bilabiale sorda intervocalica in opra D 'opera', accanto alle forme di S (h)uovra A63, B23, D154, E104, huovre A270 e passim, che presentano la sonorizzazione e la spirantizzazione della consonante (UV ovra, ovre, $\mathrm{O}$ ovre). La spirantizzazione della bilabiale sorda in nesso con liquida (anche secondario) è regolare sia in $\mathrm{S}$ che in $\mathrm{V}, \mathrm{U}$ e $\mathrm{O}$ (è un tratto genericamente settentrionale, cfr. Rohlfs 1966-69: § 260): cavra B260 'capra'; sovra A40 e passim 'sopra'; savrai D47 'saprai', ecc. Il mantenimento della sorda si riscontra unicamente in opra, forma latineggiante o più probabilmente toscana (vista la caduta della vocale postonica).

\subsubsection{Trattamento dell'occlusiva velare sorda intervocalica}

Si osserva l'assenza della sonorizzazione della velare intervocalica in secondo D, mentre il testo di S presenta unicamente l'esito lenito in segondo A83 e passim. Verosimilmente la sorda di secondo è spiegabile per influsso del modello latino da cui si traduceva.

\subsubsection{Affricata palatale sorda di tipo toscano come esito di $\mathbf{J}$}

L'affricata palatale è presente, almeno a livello grafico, in giudicio $\mathrm{D}$, giusti $\mathrm{F}$, accanto alle grafie latineggianti di S iusto D154, E204, G35, iusti D106, G337 e iustixia $\mathrm{G} 447$, da una parte, e la grafia çiusti D344, riconducibile alla fonetica settentrionale (esito di affricata dentale sonora, la $<\mathrm{i}>$ non corrisponde ad alcun

21 Piuttosto che dell'esito -àe $>\grave{e}$, non attestato altrove in $\mathrm{V}$, sembrerebbe trattarsi di un lapsus calami (omissione involontaria della lettera $a$ davanti a $e$ durante il processo di copia). 
suono, cfr. sopra 3.1.3). Si tratta quasi sicuramente di un toscanismo grafico dovuto alla tendenza ad aderire a un modello linguistico prestigioso, tratto presente anche altrove in S e assente in V e U: giovar B228 accanto a çiova E169 'giova' (V çoar, çoa, U çoar); gioventù A98 (OUV çoventù); giudegar D136 accanto a ç(i)udegar D18, D60, D104, D204 'giudicare' (V çuigar); giudei D246 accanto a çudeo D205 e çudei F121, G144 (V çuei, çue'); giuogo B189, E309 accanto a çoco E193 'gioco' (UV çogo); giurà B314 'giurato' (UV çurà); giamai E155 'mai' accanto a çamai A84 e passim (UV çamai, O çamai, çama'), ecc. Tuttavia, per la parola giusto in S viene sempre scelta la grafica etimologica iusto, iusti.

\subsubsection{Esito di c e G + vocale palatale}

Le rubriche presentano l'esito settentrionale di affricata dentale sorda in dolçe $\mathrm{C}$, e sonora in çente E. Verosimilmente anche le grafie celeste A e cità B corrispondono all'esito di affricata dentale sorda (cfr. nota 18), e l'omissione della cediglia è dovuta all'influenza della grafia latina; nel testo di S le grafie dolçe A171 e passim e çente A55 e passim si alternano con dolce C76, G87 (ma potrebbe trattarsi solo di una dimenticanza della cediglia) e giente A74 e passim.

\subsubsection{Esito di c + vocale velare}

Nella forma ciascun D si osserva la palatalizzazione della velare iniziale dovuta all'origine galloromanza del termine - il francese antico chascun (Rohlfs 1966-69: $\S 501$ ); la forma è presente anche nel testo dei componimenti (A45, A109, B179, B205, B274, D299), accanto a forme che rappresentano degli incroci tra la succitata forma toscaneggiante ciascun e la forma settentrionale çascaun (è questa la forma di V, U e O), sempre con palatalizzazione della velare iniziale: ciaschaun A155, F67; çaschaun A192; ciascadun A97; è attestato anche il toscanismo ciascheduna A9722; tutte queste grafie devono corrispondere alla pronuncia settentrionale (l'affricata [ts]). La palatalizzazione della velare davanti ad $a$ è presente anche nei gallicismi ciaramelle A116 < CALAMELLAE 'piva a due canne una delle quali è congiunta con l'otre che emette l'aria, mentre l'altra serve per modulare il suono' (TLIO: s. v. ciaramella), attraverso il fr. ant. chalemelle (V celamelli, U caramelle, O çalamella), çanbre < CAMERAE A67 (V çambre, OU çanbre) (Monteverdi 1930: 20; Contini 1960: 629, ecc.). Questo fenomeno non è molto caratteristico dal punto di vista delle particolarità delle rubriche di $\mathrm{S}$, in quanto non è esclusivo di questo manoscritto, ma presente anche in $\mathrm{V}, \mathrm{U}$ e $\mathrm{O}$.

22 Le forme ciascadun e ciascheduna sono interpretabili come incroci di caduno $<$ CATA-UNU(M) $<$ gr.

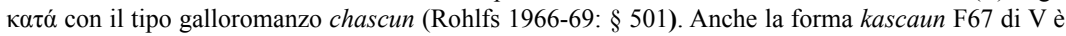
probabilmente un incrocio di caduno e chascun. 


\subsubsection{Esito di $\mathrm{SC}+$ vocale anteriore}

Si osserva l'assibilazione settentrionale (l'esito di sibilante dentale sorda da SC) in nasimento E 'nascita'. Altrove S presenta l'oscillazione tra le forme assibilate e quelle che aderiscono al modello grafico latino e toscano: cognoser C290 'conoscere' e cognosi G351 'conosci' accanto a cognoscier E41, G320 'conoscere', cognoscio A196 'conosco', cognosci E65 'conosci', cognoscie G177 'conosce', cognoscì B279 'conoscete' (V cognosro, cognoxo, cognosci e cognosì, U cognose, cognosi); nasù B243 'nato' (V naxù, U nasù) accanto a nascier B264, F228, nasciè G202 'nacque' (V nasro, nasè contro U nascer); resusitar D73 'risuscitare' accanto a resuscitar E284, E286 (V suscitar, resusitar); asende F78, sendo A162 'ascende' e asend(e)rà A24, F82 'ascenderà' accanto ad asciende F4 (V ascendo, ascendrà, $\mathrm{U}$ ascende, ascendrà).

\subsubsection{Esito di TJ}

Come il resto del codice $\mathrm{S}$, le rubriche presentano due esiti - forse solo grafici - del nesso TJ, ovvero l'affricata dentale sorda, resa tramite $<c ̧>0<c ̧ c ̧>$ in belleçça A, allegreçe A, bructeça $\mathrm{B}$, oppure tramite la grafia latineggiante $<\mathrm{ci}>$ in operacion $\mathrm{C}$, oracion $\mathrm{G}$, e la sibilante dentale sorda nel gallicismo dispresiato $\mathrm{E}$ 'disprezzato' $<$ fr. ant. despriser (TLIO: s. v. dispregiare). Le voci del verbo dispresiare (anche nella variante grafica disprexiare) si riscontrano in alcuni testi veneti, tra i quali la veneziana Cronica de li imperadori (Ceruti 1878: 182 e 233), i Quatro Evangelii del veneziano Jacopo Gradenigo (Gambino 1999: 86), il Lucidario veronese (Donadello 2003: 36 e 101), ecc., e in un testo emiliano, ovvero il commento alla Commedia del bolognese Iacomo della Lana (Volpi 2009: 164 e passim). L'esito di sibilante dentale è attestato in $\mathrm{S}$ sempre in vocaboli di provenienza galloromanza: malvaxii $\mathrm{F} 8 \mathrm{e}$ malvasità D178 'malvagi' (V malvas, malvasità) da *MALIFATICU(M) 'malaugurato' attraverso fr. ant. malvais o prov. ant. malvatz (DELI: s. v. malvagio); palasii B302 'palazzi' (UV palasi); si tratta di forme tipiche delle scriptae venete antiche (cfr., ad esempio, Stussi 1965: LV n. 64).

\subsubsection{Epentesi di $r$ negli avverbi in -mente}

Le rubriche sono caratterizzate dall'assenza dell'epentesi di $r$ nelle forme avverbiali in -mente: magnificamente $\mathrm{F}$, ultimamente $\mathrm{G}$, e questa situazione coincide con il resto del testo di S. L'epentesi, caratteristica degli antichi dialetti lombardo, veronese e veneziano (Ascoli 1873: 459; Ineichen 1966: 381; Rohlfs 1966-60: § 333; Sattin 1986: 97; Gambino 1996: 241, ecc.), appare invece sporadicamente in V e in U (ma non in $\mathrm{O}$ ): V duramentre B207, fermamentre F157, orribelmentre B209, ecc.; U 
enprimamentre A41 'all'inizio, in primo luogo', solamentre A86 (cfr. S duramente B207, D135; fermamente F157; inprimamente A41; oribelmente B209; solamente A86). Le poche attestazioni di questo fenomeno in $\mathrm{S}$ risalgono sicuramente a piani alti della tradizione manoscritta: soletamentre $\mathrm{C} 109$ 'soltanto' accanto a soletamente C160; devotamentre D6 accanto a devotamente C6, F235, D120, F221.

\subsubsection{Metatesi}

La forma ternitade $\mathrm{G}$ 'trinità', che si riscontra anche in $\mathrm{O}$ (ternità $\mathrm{A} 154)$, ma non in $\mathrm{V}$ e U (i codici avevano sempre trinità), è l'unica forma di questa parola impiegata in $\mathrm{S}$ (se escludiamo il latinismo puro trinitas G363): ternità A154, G294, G332, G345, ternitade G329 e Ternitatem Gtit345. L'esito ternitade può essere interpretato come metatesi con la successiva apertura della vocale davanti a $r$, oppure come scambio di radicale (Corti 1962: LIX), oppure ancora come "sincope o indebolimento vocalico tra muta e $r$ " (Borgogno 1980: 39).

\subsection{Morfologia}

\subsubsection{Metaplasmo di declinazione}

Il passaggio dalla terza declinazione latina alla prima si riscontra nell'aggettivo infernala B. La sostituzione della desinenza ambigua (quella della terza declinazione) con un'altra più 'trasparente' (quella della prima o della seconda declinazione, a seconda del genere del vocabolo) è un fenomeno molto frequente nei volgari settentrionali e soprattutto in veronese (Rohlfs 1966-69: § 353) e attestato ampiamente in V e U (per i metaplasmi di genere maschile - di tipo flumo, fanto, sango - è da prendere in considerazione anche la spiegazione fonetica del fenomeno, ovvero il reintegro della -e caduta con $-o$, cfr. sopra nota $15^{23}$ ). S, come anche $\mathrm{O}$, dimostra una notevole resistenza a questo tratto, cosa che si evince dal confronto delle sue lezioni con quelle di V e U: breve E111 contro V breva; brievemente G85 contro V brevoment; crudelle B223, B321 contro UV crudela; forte B313 contro V forta; grieve E226, E309, G120 contro V grevo e greva; lucente A93 'luminoso' contro V lucento (ma OU lucente); puçolente B90, B148, C242, E74, E106 contro UV puçolento; sangue B128 contro V sango, ecc. Tuttavia, S presenta anche brevo E231 accanto a breve E111; fragila E112; grando B142, D294, G235 e granda A137, B205, B313 accanto a grande A92 e passim; quala C239 accanto a qual

23 A parte va considerato l'aggettivo grando / granda, attestato in tutto il settentrione e non solo nelle zone di caduta di vocale finale, per cui in questo caso il metaplasmo va visto come causa e non come effetto. 
A11 e passim, redolenta A177, C239 'profumata, aromatica' accanto a redolente A109, ecc. La forma infernala è da annoverare tra i tratti marcatamente dialettali (anche se genericamente settentrionali e non caratteristici di un'area municipale in particolare) presenti nelle rubriche di S.

Un altro metaplasmo riscontrato nelle rubriche di S è loldo 'lode': la parola ha effettuato il passaggio dalla terza declinazione alla prima e ha cambiato anche genere, da femminile diventando maschile. Nel titolo del componimento F il termine è attestato al plurale: Delli loldi e nobilitade [...] li qualli... F; che il singolare sia quasi sicuramente loldo, lo si può affermare con buon margine di sicurezza grazie ai riscontri di questa forma del singolare in vari testi veneti (soprattutto veneziani): i Vangeli in veneziano (Gambino 2007: 15 e passim), la vita dei santi Pietro e Paolo in veneziano (Brusegan Flavel 2005: 67), la redazione veneta della Navigazione di San Brandano (Grignani 1975: 100 e 116), ecc., ed emiliani: le arringhe di Matteo dei Libri (Vincenti 1974: 232 e passim), il laudario dei Battuti di Modena (Elsheikh 2001: 23), un inventario modenese (Bertoni 1913: 160). Tuttavia, si riscontra anche l'esito lolde (sempre maschile): sempre se de' reportare lo lolde del maiore inançi (Vincenti 1974: 232, attestazione unica nel corpus dell'OVI). Esiste anche la forma lolde femminile - quella che è logico aspettarsi come continuatore di LAUS, LAUDIS - che conta, tuttavia, solo un'attestazione al singolare: la lolde reportata per altri (Vincenti 1974: 233) e qualche attestazione al plurale: le suo' lolde in un testo veneziano (Brusegan Flavel 2005: 67 e 75), le lolde in un testo ferrarese (Stella 1968: 212); tuttavia, lo stesso componimento veneziano presenta anche li loldi (Brusegan Flavel 2005: 67 e 74), forma attestata anche in altri testi di area venetoemiliana (Levi 1917: 9; Galasso 1937: 90; Vincenti 1974: 285 e 305; Verlato 2009: 62 e 69). Nel nostro caso è abbastanza improbabile che si tratti di lolde femminile, visti l'articolo li e la concordanza con li qualli della proposizione relativa.

\subsubsection{Pronome dimostrativo quella con valore di pronome personale di terza persona femminile}

Il pronome è attestato in questa posizione sintattica due volte, nelle rubriche dei primi due componimenti, e probabilmente il suo uso con questa funzione è dovuto alla traduzione di una struttura grammaticale latina (di quella è usato per rendere eius).

\subsubsection{Aggettivo possessivo sua}

L'uso del possessivo sua della rubrica E discosta dalla lingua di V e di U (i codici presentavano sempre soa) e in certa misura anche di S, il quale presenta per il femminile singolare soa A11 e passim e sua A23 e passim (la prima forma prevale 
sulla seconda). La scelta della forma sua sembra dettata, come in molti altri casi, dall'esigenza di rendere la lingua 'più corretta', attingendo ai modelli toscano o latino, o semplicemente dal fatto di tradurre dal latino.

\subsubsection{Forme e desinenze della terza persona del plurale}

I morfemi della terza plurale sono sconosciuti a $\mathrm{V}$, a U e all'archetipo, nonché quasi del tutto assenti nella redazione di $\mathrm{S}$, mentre nelle rubriche del codice colombino leggiamo sono ponidi B 'sono puniti', ricieveno F 'ricevono', ricieverano F 'riceveranno'. La terza singolare con valore di plurale nei titoli non ricorre mai, invece nel testo del componimento è la regola: quellor che 'l bon Jesù non ama A12 'coloro che il buon Gesù non amano'; lli santi ne parla e sì ne favella B8 'gli santi ne parlano e ne favellano', ecc. Due delle quattro eccezioni riscontrate nel testo principale non sono interpretabili univocamente come forme della terza plurale, in quanto si tratta di due tituli (segnalati qui con le parentesi tonde) e non di desinenze trascritte in forma piena: enpie(n) B216 'riempiono' (V emplo); dixo(n) A29 ‘dicono' (V diso); i restanti due casi sono poseseno G318 'potessero' (V pos') e l'errore eran B20 per enançi 'prima' (V enançi, U anti).

L'assenza nei titoli delle forme di terza singolare con valore di terza plurale si inscrive nel quadro delle tendenze 'normalizzanti' (in direzione del toscano), mirate a eliminare tratti spiccatamente dialettali.

\subsubsection{Assenza della forma analitica del futuro}

Le forme analitiche del futuro (il tipo HABERE + infinito) sono presenti frequentemente nell'archetipo e attestate ogni tanto anche in S, accanto alle forme sintetiche: v'ò dir A40 'vi dirò'; à çiudegar D18, D60 'giudicherà'; à ligar B78 'legheranno'; à vegnir $\mathrm{B} 171$ 'verrà', ecc., accanto a meterà $\mathrm{F} 82$ 'metterà'; piaxerà G279 'piacerà', ecc. In $\mathrm{S}$ ha luogo la riduzione delle forme di futuro separabili, come illustrano le seguenti coppie di varianti: S arderà] V à ardro D14; S consumerà] V [à] consumar D14; S sonerà] V à sonar D74. Si registra anche un caso inverso: S à trovar] V trovarà E96.

Nelle rubriche si riscontrano solo forme sintetiche: avrà $\mathrm{D}$, ricieverà $\mathrm{D}$, ricieverano $\mathrm{D}$, un altro tratto 'normalizzante' che fa pensare a una datazione piuttosto tarda del volgarizzamento di queste rubriche (la seconda metà del Trecento).

\subsubsection{La preposizione in de con il significato di 'in'}

È attestata tre volte: in del core $\mathrm{C}$, in del qual $\mathrm{D}$, in del presente mondo $\mathrm{E}$, e fa parte delle innovazioni linguistiche delle rubriche di $\mathrm{S}$ rispetto al testo principale. 


\subsection{Lessico}

3.4.1. È da notare il termine piangolente, un aggettivo derivante dal participio presente con l'infisso in laterale; il suo significato è 'doloroso, che comporta pianto'. Il vocabolo è attestato in tre testi di area veneto-emiliana: piangolenta nei volgarizzamenti veneti trecenteschi dell'Ars amandi e dei Remedia amoris di Ovidio (Lippi Bigazzi 1987, I: 491), piangolenti nel laudario dei Battuti di Modena (Elsheikh 2001: 303), plangolent nei Quatro Evangelii in veneto di Gradenigo (Gambino 1999: 49).

3.4.2. L'avverbio dapoi $\mathrm{G}$ 'poi, dopo, successivamente' non ricorre mai altrove in $\mathrm{S}$, che presenta invece poi $\mathrm{B} 64$ e passim (V po'), po' B57 e apresso G229. La parola conta numerose attestazioni in testi di varia provenienza (settentrionali e non solo).

\section{Conclusioni: Venezia o Bologna?}

I titoli del codice S si discostano dal testo principale in quanto non contengono fenomeni riconducibili all'ascendente veronese del manoscritto, nonché per la maggiore moderazione nell'uso dei tratti settentrionali in generale (come la lenizione delle occlusive labiali e velari intervocaliche o la terza singolare dei verbi con valore di plurale); la tendenza generale consiste nell'introdurre un certo numero di tratti di matrice dotta, latina (come le grafie etimologiche, semidotte e ipercorrette, la resistenza al dittongamento spontaneo, il mantenimento o la restituzione delle occlusive intervocaliche sorde) o toscana (come le grafie giusti o ciascun che si richiamano al vocalismo toscano, ancora le occlusive intervocaliche sorde, o le forme specifiche della terza persona del plurale, sia dal punto di vista morfologico che da quello fonetico). I tratti latineggianti sembrano essere riconducibili al volgarizzatore delle rubriche, mentre di quelli toscaneggianti possono essere responsabili anche copisti posteriori.

Tuttavia, nonostante una certa patina allo stesso tempo latineggiante e toscaneggiante, le rubriche di S contengono anche dei fenomeni di matrice dialettale settentrionale: il raddoppiamento grafico non etimologico di $l$ in Babillonia e qualli e lo scempiamento della doppia in devotisima, l'esito $a u>o l$ in loldi, il dittongo in fiuol, il passaggio $\mathrm{U}>o$ in protonia in ponidi (anche se non è un tratto esclusivamente settentrionale), l'apocope di -e e - o dopo nasale, la dentale sonora intervocalica in pecadi, ponidi ecc., il dileguo della dentale davanti a liquida in mare, l'affricata dentale in çente e dolçe, la sibilante dentale in dispresiato e nasimento, la metatesi (o scambio di 
radicale) in ternitade, i metaplasmi infernala e loldi, il lessema piangolente. Tali tratti rendono plausibile la provenienza veneziana delle rubriche (oltre ai tratti elencati, con il volgare di Venezia sono compatibili l'esito Dio e la forma integra del femminile plurale nobilitade); tuttavia non si può neanche scartare l'ipotesi della loro localizzazione in Emilia, probabilmente a Bologna: è tipica della scripta bolognese la restituzione delle vocali finali dopo liquida, non è infrequente la restituzione delle dentali intervocaliche nella forma sonora, le voci loldo/lolde e piangolente sono attestate anche in testi emiliani antichi, i tratti genericamente settentrionali (assibilazione, metaplasmi, ecc.) si riscontrano in emiliano come in veneto; inoltre, la presenza di non pochi toscanismi rende l'origine bolognese di questi titoli non improbabile, visto che, come nota Maria Corti, "Bologna [...] ci tramanda per lo più una lingua strutturata già in partenza sul latino e sul toscano, tesa a liberarsi dagli elementi dialettali" (Corti 1962: X). Purtroppo non ci sono sembrati individuabili sufficienti elementi linguistici per dirimere tra le due localizzazioni alternative.

\section{Bibliografia}

Andreose, A. (2002): "La prima attestazione della versione VA del Milione (ms. 3999 della Biblioteca Casanatense di Roma). Studio linguistico”, Critica del testo $\mathrm{V} / 3,655-668$.

Arcangeli, M. (1990): “Per una dislocazione tra l'antico veneto e l'antico lombardo (con uno sguardo alle aree contermini) di alcuni fenomeni fonomorfologici", L'Italia dialettale LIII, 1-42.

Ascoli, G. I. (1873): “Saggi ladini”, Archivio glottologico italiano I, 1-573.

Badas, M. (2009): La 'Legenda de Santo Stadi', Franceschino Grioni (RomaPadova: Antenore).

Barana, E. (1921): Giacomino da Verona, 'La Gerusalemme celeste'e 'La Babilonia infernale', secondo la lezione dei quattro codici conosciuti (Verona: La tipografia veronese).

Bertoletti, N. (2005): Testi veronesi dell'età scaligera (Padova: Esedra).

Bertoni, G. (1913): “Un inventario modenese del sec. XIV (1374)”, Atti e Memorie della R. Deputazione di Storia Patria per le provincie modenesi V, VII, 147-60.

Borgogno, G.B. (1980): "La lingua dei dispacci di Filippo della Molza, diplomatico mantovano della seconda metà del secolo XIV", Studi di grammatica italiana IX, 19-171. 
Brusegan Flavel, E. (2005): "La 'Legenda di glorioxi apostoli misier sen Piero e misier sen Polo’ (codice Venezia, B.M.C. Correr 1497)”, Quaderni veneti XVI, 7-108.

Burgio, E. (1995): 'Legenda de misier Sento Alban'. Volgarizzamento veneziano in prosa del XIV secolo (Venezia: Marsilio).

Caprettini, G.P. (1971): “-o atona finale nella 'Passione veronese'”, Atti del VII Convegno del Centro per gli studi dialettali italiani, 157-161 (Torino: Stamperia Editoriale Rattero).

Ceruti, A. (1878): “Cronica deli imperadori romani”, Archivio glottologico italiano III, 177-243.

Contini G. (1938): "Un manoscritto ferrarese quattrocentesco di scritture popolareggianti", Archivum Romanicum XXII, 281-319.

Contini, G. (1960): Poeti del Duecento, 2 voll., vol. II, 625-650 (Milano-Napoli: Ricciardi).

Cornagliotti, A. (1979): "Un volgarizzamento del 'Transitus Pseudo-Josephi de Arimathea' in dialetto veronese", Atti della Accademia delle Scienze di Torino, CXIII, 197-217.

Cortelazzo, M., Zolli, P. (1999): Dizionario Etimologico della Lingua Italiana [= DELI] (Bologna: Zanichelli).

Corti, M. (1960): "Emiliano e veneto nella tradizione manoscritta del 'Fiore di virtù", Studi di filologia italiana XVIII, 29-68.

Corti, M. (1962): Vita di S. Petronio. Con un'appendice di testi inediti dei secoli XIII e XIV (Bologna: Commissione per i testi di lingua).

Corti, M. (1966): "Una tenzone poetica del sec. XIV in veneziano, padovano e trevisano", en Branca, V. / Padoan, G. (edd.): Dante e la cultura veneta, 129142 (Firenze: Olschki).

Delcorno, C: (1998): "Produzione e circolazione dei volgarizzamenti religiosi tra Medioevo e Rinascimento", in Leonardi, L. (ed.): La Bibbia in italiano tra Medioevo e Rinascimento (Firenze: SISMEL, Edizioni del Galluzzo), 3-22.

De Luca, G. (1963): Letteratura di pietà a Venezia dal '300 al '600 (Firenze: Olschki).

Donadello, A. (2003): Lucidario. Volgarizzamento veronese del XIV secolo (RomaPadova: Antenore).

Elsheikh, M. S. (2001): Il Laudario dei Battuti di Modena (Bologna: Commissione per i testi di lingua). 
Ferguson, R. (2005): “Alle origini del veneziano: una koiné lagunare”, Zeitschrift für Romanische Philologie CCXXI, 476-509.

Folena, G. (1991): Volgarizzare e tradurre (Torino: Einaudi).

Frati, C. (1911): "Dicerie volgari del sec. XIV aggiunte in fine del 'Fior di virtù", Studi letterari e linguistici dedicati a Pio Rajna nel quarantesimo anno del suo insegnamento, 325-335 (Firenze: Ariani).

Frati, C., Segarizzi, A. (1909-1911): Catalogo dei codici marciani italiani, 2 voll., vol. II (Modena: Ferraguti).

Galasso, M. (1937): Il Tristano Corsiniano (Cassino: Le Fonti).

Gambino, F. (1996): "Ibridismo linguistico in un poema veneziano di fine '300: 'Gli quatro Evangelii concordati in uno' di Jacopo Gradenigo”, Italia dialettale LIX, 211-298.

Gambino, F. (1999): Gli Quatro Evangelii concordati in uno di Jacopo Gradenigo. Introduzione, testo e glossario (Bologna: Commissione per i testi di lingua).

Gambino, F. (2007): I Vangeli in antico veneziano: ms. Marciano it. I 3 (4889) (Roma-Padova: Antenore).

Ghinassi, G. (1965): "Nuovi studi sul volgare mantovano di Vivaldo Belcazer", Studi di filologia italiana XXIII, 19-172.

Grignani, M. A. (1975): Navigatio Sancti Brendani. La navigazione di San Brandano (Milano: Bompiani).

Ineichen, G. (1966): El libro agregà de Serapiom. Volgarizzamento di frater Jacobus Philippus de Padua, 2 voll., vol. II (Venezia-Roma: Istituto per la collaborazione culturale).

Levi, E. (1917): Il libro dei cinquanta miracoli della Vergine (Bologna: Romagnoli - Dall'Acqua).

Lippi Bigazzi, V. (1987): I volgarizzamenti trecenteschi dell'Ars amandi' e dei 'Remedia amoris', 2 voll., vol. I (Firenze: Accademia della Crusca).

May, E. I. (1930): The 'De Jerusalem celesti' and the 'De Babilonia infernali' of Fra Giacomino da Verona (Firenze: Le Monnier).

Monteverdi, A. (1930): “La leggenda di Santo Stady di Franceschino Grioni”, Studj Romanzi XX, 1-199.

Mussafia, A. (1864): Monumenti antichi di dialetti italiani (Vienna: Academia delle Scienze di Vienna). 
Ozanam, F. (1850): Documents inédits pour servir a l'histoire de l'Italie depuis le VIIIeme siècle jusqu'au XIIeme, avec des recherches sur le moyen âge italien (Paris: Lecoffre).

Pellegrini, G.B. (1966): "La posizione del veronese antico", en Branca, V. / Padoan, G. (edd.): Dante e la cultura veneta, 95-107 (Firenze: Olschki).

Pellegrini, G.B. (1977): "Dialetti veneti antichi”, en Id., Studi di dialettologia e filologia veneta, 33-88 (Pisa: Pacini).

Raugei, A.M. (1984): La navigazione di San Brendano. Versione italiana del ms. Bologna, Bibl. Univ. 1513 (Fassano di Puglia: Schena).

Riva, F. (1953): Storia dell'antico dialetto di Verona secondo i testi in versi (dal sec. XIII al sec. XVII): Fonetica, Estratto dagli Atti dell'Accademia di Agricoltura, Scienze e Lettere di Verona, Serie VI, Volume III, anno 1951-52 (Verona: La Tipografia Veronese).

Rohlfs, G. (1966-1969): Grammatica storica della lingua italiana e dei suoi dialetti, 3 voll. (Torino: Einaudi) [si cita per paragrafo].

Sattin, A. (1986): "Ricerca sul veneziano del sec. XV (con edizione di testi)", L'Italia dialettale XLIX, 1-172.

Segre, C. (1953): Volgarizzamenti del Due e Trecento (Torino: Tipografia torinese).

Stella, A. (1968): “Testi volgari ferraresi del secondo Trecento", Studi di filologia italiana, XXVI, 201-310.

Stussi, A. (1965): Testi veneziani del Duecento e dei primi del Trecento (Pisa: NistriLischi).

Tesoro della Lingua Italiana delle Origini, http://tlio.ovi.cnr.it/TLIO/ [= TLIO].

Tomasin, L. (2004): Testi padovani del Trecento. Edizione e commento linguistico (Padova: Esedra).

Tomasoni, P. (1973): "Per una storia dell'antico Trevigiano", Studi di grammatica italiana III, 155-206.

Verlato, Z. (2002): "L'inedita redazione veronese di un 'Contrasto tra Cristo e il diavolo", Quaderni veneti XXXVI, 9-42.

Verlato, Z. (2009): Le vite di santi del codice Magliabechiano XXXVIII.110 della Biblioteca Nazionale Centrale di Firenze. Un leggendario volgare trecentesco italiano settentrionale (Tübingen: Niemeyer).

Vincenti, E. (1974): Matteo dei Libri. Arringhe (Milano-Napoli: Ricciardi). 
Volpi, M. (2009): Iacomo della Lana, Commento alla 'Commedia', 4 voll. (Roma: Salerno Editrice).

Zvonareva, A. (2012): "Un caso di ibridismo linguistico tra Veneto, Emilia e Toscana nel Trecento: qualche osservazione sul codice 7-1-52 della biblioteca Colombina di Siviglia", Atti dell'incontro 'Filologia románica hoy' (Madrid, novembre 2011) [in corso di stampa]. 
